\title{
TriBeam Tomography for 3D Data Acquisition
}

McLean Echlin ${ }^{1}$, Andrew Polonsky ${ }^{1}$, Toby Francis ${ }^{1}$, Will Lenthe ${ }^{2}$, Mike Titus ${ }^{3}$, Alessandro Mottura ${ }^{4}$, Chris Torbet $^{1}$, Steven Randolph ${ }^{5}$, Aurelien Botman ${ }^{5}$, Jorge Filevich ${ }^{5}$, Remco Geurts ${ }^{6}$, Marcus Straw ${ }^{7}$ and Tresa Pollock ${ }^{1}$

${ }^{1}$ University California-Santa Barbara, San Francisco, California, United States, ${ }^{2}$ Carnegie Mellon University, Pittsburgh, Pennsylvania, United States, ${ }^{3}$ Purdue University, West Lafayette, Indiana, United States, ${ }^{4}$ Birmingham University, Birmingham, England, United Kingdom, ${ }^{5}$ Thermo Fisher Scientific, Hillsboro, Oregon, United States, ${ }^{6}$ Thermo Fisher Scientific, Eindhoven, Noord-Brabant, Netherlands, ${ }^{7}$ Applied Physics Technology, McMinnville, Oregon, United States

A range of instruments have been developed for the removal and deposition of material in an electron microscope vacuum chamber, including gallium source focused ion beams (FIBs), plasma FIBs (Xe, O, $\mathrm{N}$, and Ar charged species), broad ion beams (BIBs), ultramicrotomes, and more recently nanosecond, picosecond and femtosecond lasers. Depackaging and failure analysis of integrated circuits and packaged electronic devices are applications enabled by in situ material removal, however serial sectioning for the reconstruction of high resolution 3D tomographic data has also become critical for material science. Although serial sectioning techniques are inherently destructive making them unsuitable for time-resolved (4D) data collection, compared to the $\mathrm{x}$-ray techniques they can provide rich, high resolution multimodal information particularly from strongly absorbing materials. Furthermore, serial sectioning techniques have been effectively used in combination with 4D x-ray grain mapping techniques to capture grain growth evolution, providing a high resolution final dataset with prior time resolved data [1,2].

The TriBeam microscope was developed specifically for 3D data acquisition by leveraging a femtosecond laser to provide fast, low damage material removal that is effective across most material classes (metals, ceramics, composites, soft materials, and bio-materials). The TriBeam [3,4] is based on a FIB-SEM microscope platform that has been modified to incorporate a femtosecond laser, an optics beamline, beam steering and alignment diagnostic equipment, and a shutter to protect the detectors and electron beam pole piece during in situ laser ablation. An extensive codebase also exists for fully automated TriBeam system controls, which can run a serial sectioning experiment continuously for weeks on end if needed. 3D TriBeam data has been influential in understanding the solidification processes active during additive manufacturing [5] and the defects that form [6], fatigue in nickel-base superalloys [7], the mechanical behavior of titanium [8,9], and parameters critical for the for the performance of $\mathrm{W}-\mathrm{Cu}$ composites in high temperature applications [10].

Recently, an updated femtosecond enabled microscope system has been developed [11] on a Xe plasma FIB platform with variable laser beam wavelength. Laser ablation with $515 \mathrm{~nm}$ pulses reduces the spacing and amplitude of light induced periodic surface structures (LIPSS) and can be used on materials that do not absorb as readily at longer wavelengths. Furthermore, low damage cut faces have been produced in a range of soft materials, including mylar, PTFE, resin embedded brain and liver tissue [12].

In this tutorial a range of topics will be discussed, including the TriBeam experimental setup and the gathering of multimodal data, femtosecond laser-material interactions for a range of material systems [13], automation and controls for new tomography experiments, and reconstruction and visualization of 3D data. With the wealth of new 3D characterization methods available, data sharing and provenance will be 
discussed in the context of the open-sourced and collaborative software platform BisQue [14], which has simplified multi-institution research projects that utilize 3D datasets.
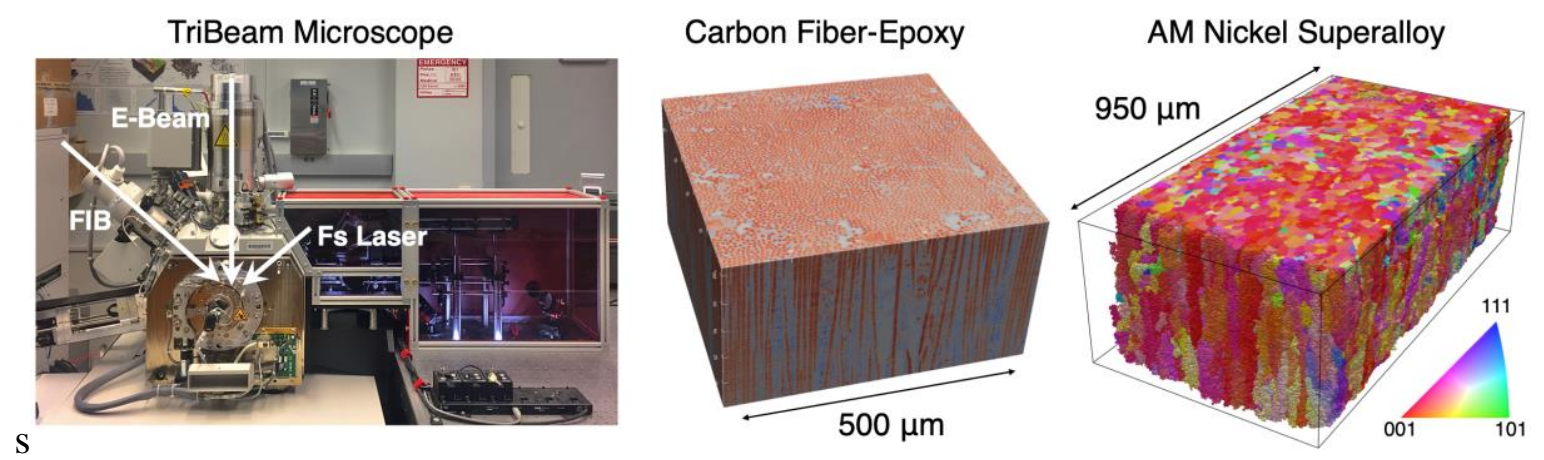

Figure 1. The TriBeam microscope combines a femtosecond laser with a FIB-SEM for in situ material ablation with limited damage for the acquisition of mm-scaled multimodal 3D data.

References

[1] W.C. Lenthe et al. J. Appl. Cryst. 48. 2015. pg 10341046. https://doi.org/10.1107/S1600576715009231

[2] A. Trenkle et al. Non-destructive evaluation of 3D microstructure evolution in strontium titanate. J. Appl. Cryst. 2020. In Press. https://doi.org/10.1107/S160057672000093X

[3] M.P. Echlin et al. Review Scientific Instruments. 83. 2012. 023701. pg 16. https://doi.org/10.1063/1.3680111

[4] M.P. Echlin et al. Mat. Char.: Tutorial Review. 100. 2015. pg 112. https://doi.org/10.1016/j.matchar.2014.10.023

[5] A.T. Polonsky et al. 3D Characterization of the Columnar-to-Equiaxed Transition in Additively Manufactured Inconel 718. Superalloys 2020. Submitted.

[6] A.T. Polonsky et al. Mat. Char.143, 2018. pg 171-181. https://doi.org/10.1016/j.matchar.2018.02.020

[7] W.C. Lenthe et al. Superalloys. 2016. pg 569-577. https://doi.org/10.1002/9781119075646.ch61

[8] K. Chatterjee et al. Acta Mat. 157, 2018. pg 21-32. https://doi.org/10.1016/j.actamat.2018.07.011

[9] M. Kasemer et al. Acta Mat. 136, 2017. pg 288-302. https://doi.org/10.1016/j.actamat.2017.06.059

[10] M.P. Echlin et al. Acta Mat. 64. 2014.pg 307-315. https://doi.org/10.1016/j.actamat.2013.10.043

[11] S.J. Randolph et al. J. Vac. Sci. Tech. B, 36.6 (2018): 06JB01. https://doi.org/10.1116/1.5047806

[12] S.J. Randolph et al. Microsc. Microanal. 25.S2 (2019): 352-

353. https://doi.org/10.1017/S1431927619002496

[13] M.P. Echlin et al. Acta Mat. 124. 2016. pg 37-46. https://doi.org/10.1016/j.actamat.2016.10.055

[14] A.T. Polonsky et al. IMMI 8.1 (2019): 37-51. https://doi.org/10.1007/s40192-019-00126-7 